\title{
Minimantle Radiation Field
}

National Cancer Institute

\section{Source}

National Cancer Institute. Minimantle Radiation Field. NCI Thesaurus. Code C53632.

A radiation field that includes the bilateral cervical, supraclavicular, and axillary lymph nodes. 